\title{
On the Feedback Transfer Effort for Rate-Adaptive Multiuser Systems
}

\author{
Anthony E. Ekpenyong and Yih-Fang Huang \\ Dept. of Electrical Engineering \\ 275 Fitzpatrick Hall \\ University of Notre Dame \\ Notre Dame, IN 46556 \\ Email: \{aekpenyo, huang\}@nd.edu
}

\begin{abstract}
The benefits of multiuser diversity for fading channels are well established in the literature. What is not well documented is the effort required to obtain this CSI information at the scheduler for a frequency division duplex (FDD) system. This paper investigates the practical aspects of the feedback transfer process for a discrete rate-adaptive multiuser system including multi-access, feedback delay and feedback channel imperfections due to fading and additive noise. We compute the optimal power and bandwidth allocated for channel estimation and show that tradeoffs exist between these resources and the number of users depending on the feedback delay. It is also shown that quantization and feedback errors may increase the outage region as the number of users increases. This in effect reduces the multiuser diversity gain expected in ideal feedback channels.
\end{abstract}

\section{INTRODUCTION}

Channel state information (CSI) is important for multiuser systems to facilitate user scheduling in addition to resource allocation at the transmitter. It has been shown that independence among the channels linking the base transceiver station (BTS) and a group of users leads to multiuser diversity gain [1], [2], whereby the BTS transmits to the user with the best channel. Similarly to any channel-aware transmitter, CSI acquisition at the scheduler is a limiting factor to the benefits provided by multiuser diversity, particularly for frequency division duplex (FDD) systems. For example, in the downlink of a FDD cellular system, the users send their CSI estimates to the BTS on a bandwidth-, interference-, and delay-constrained reverse link, which is also degraded by fading and additive noise.

The aim of this paper is to study the effects of feedback delay, feedback errors, and multi-access issues on the feedback transfer process for a discrete rate-adaptive multiuser system. Our contributions are as follows: for a given feedback delay, the power allocation and bandwidth reserved for channel estimation are optimized. It is observed that less power is required for training as the number of users increases. Secondly, by describing how feedback delay depends on the number of users to avoid multi-access interference (MAI), it would be seen that multiuser systems are limited by mobility, which differs from the constant delay assumption in [3]. Finally, the performance degradation caused by quantization and feedback errors is investigated, and we show how the channel fading characteristics could be used to mitigate this performance degradation.

Research into feedback transfer for multiuser systems has focused on the impact of quantization [4], and on the bandwidth constraint of the reverse channel [5], [6] but possible MAI was not explored. This MAI problem was partly addressed in [7] where multiple thresholds are employed so that with high probability only one user may need to send back its CSI information. However, this scheme requires polling between the BTS and the users, and the delay may not be practical for time-selective fading channels. Moreover, user identification at the BTS implies that the feedback information is proportional to the number of users. Here, we consider an adaptive discrete rate system with simultaneous feedback transmission employing spreading codes to avoid MAI. When the system takes into account the practical constraints of quantization and feedback errors, we show that these errors introduce an outage region, which increases with the number of users.

The system is described in Section II, while Section III analyzes the system performance for noisy CSI feedback. Section IV presents numerical results on the effect of prediction, quantization, and feedback errors, and conclusions are given in Section V. In the paper, the subscripts $f$ and $r$ refer to the forward and reverse channels respectively, while $A \sim \mathcal{C N}\left(\mu, \sigma^{2}\right)$ defines $A$ as a Gaussian random variable with mean $\mu$ and variance $\sigma^{2}$.

\section{Problem Formulation}

Consider a time division multiple access (TDMA) downlink system with a group of $K$ active users served by the BTS, i.e., the BTS has data to transmit to each of the $K$ users. The paths between the BTS and the users are independent and identically distributed, time-selective flat fading channels, where $h_{k} \sim \mathcal{C N}(0,1)$ for $k=1, \ldots, K$. As such, fairness issues are not considered in this paper, and the scheduler selects the user with the best channel power [2]. For the case of non-identical users, such as when the users are at different distances from the BTS, a fair scheduling scheme such as in [3] can be used. The BTS employs frame-based transmission of $L$ symbols, where the first symbol is broadcast to all users to aid in channel estimation and the rest of the frame is used for transmission to the scheduled user. Each user predicts its own 
channel over a time duration that includes processing delay at both the user and the BTS, and also feedback transmission time. Based on the predicted states the BTS selects the best user and its desired rate from a set of $N$ QAM constellations, $\mathcal{M}=\left[M_{0}, \ldots, M_{N-1}\right]$. Denoting the SNR for the $k_{*}$ th user as $\gamma_{k_{*}}$, this user is transmitted to with rate $i$ only if

$$
\gamma_{k_{*}}=\max _{k} \gamma_{k} \text { and } t_{i} \leq \gamma_{k_{*}}<t_{i+1} \quad i=0,1, \ldots, N-1,
$$

where $t_{0}=0, t_{N}=\infty$ and $\left\{t_{i}\right\}_{i=1}^{N-1}$ is the set of switching thresholds corresponding to the transmission mode set $\mathcal{M}$. Similarly to [8], the thresholds are optimized subject to an average BER constraint. At each user, the predicted CSI is quantized to $b=\log _{2} N$ bits and is transmitted to the BTS over a reverse channel subject to fading and additive noise. For simplicity the feedback channel imperfections can be characterized by a crossover probability matrix $Q=\left[q_{i, j}\right]$, where $q_{i, j}$ denotes the probability that the $i$ th transmission mode is selected when the $j$ th mode is optimal [9]. The effects of quantization and feedback error on the scheduling process would be explored later in the paper but first the estimation process is described.

\section{A. Channel Estimation}

Channel estimation is typically carried out at the receiver for symbol detection (interpolation) and for CSI feedback to the BTS (prediction). To focus on the impact of imperfect CSI on the scheduler, we assume perfect CSI for symbol detection at the selected user. It is straightforward (see, e.g., [10]) to include the effect of channel interpolation error. For any user the received signal vector for the $m$ th frame is given by

$$
\mathbf{y}(m)=\left\{\begin{array}{l}
\sqrt{\mathcal{P}_{t}} h(m ; 0) x_{t}(m)+n_{f}(m ; 0) \\
\sqrt{\mathcal{P}_{d}} h(m ; l) x_{d}(m ; l)+n_{f}(m ; l),
\end{array}\right.
$$

for $l=1, \ldots, L-1$. $\mathcal{P}_{t}$ and $\mathcal{P}_{d}$ denote the training and data powers respectively, $n_{f} \sim \mathcal{C N}(0,1)$ is the additive noise and the training symbol $\left(x_{t}\right)$ and data symbols $\left(x_{d}\right)$ have unit power. Since in adaptive transmission there is a notransmit region, $0 \leq \gamma_{k}<t_{1}$, the actual average data power is $\overline{\mathcal{P}}_{d}=\int_{t_{1}}^{\infty} \mathcal{P}_{d}(y) f_{\gamma}(y) d y$, where $f_{\gamma}(y)$ is the probability density function (pdf) of the SNR. Hence, the total average power per channel use is defined as $\mathcal{P}_{f}=\mathcal{P}_{t}+\overline{\mathcal{P}}_{d}$. Let $\alpha$ be the fraction of the total average power in a block that is allocated for training. Then,

$$
\mathcal{P}_{t}=\alpha L \mathcal{P} \text { and } \overline{\mathcal{P}}_{d}=(1-\alpha) \frac{L}{L-1} \mathcal{P} .
$$

For equal power allocation, $\alpha=1 / L$. It is assumed that the delay, $\tau$ is a multiple of the frame length, i.e., $\tau=D L T_{s}$, where $T_{s}$ is the symbol duration and $D$ is a positive integer [10]. Clearly, within a block, $\hat{h}(m ; L-1)$ is the worst channel estimate, thus, it can be used as an estimate for the entire block. Collecting $J$ received training symbols into an observation vector $\mathbf{y}_{t}(m)=[y(m ; 0), y(m-1 ; 0), \ldots, y(m-J+1 ; 0)]$, the linear minimum mean square error variance of the channel is given by $\sigma_{\tilde{h}}^{2}=1-C_{h y} C_{y y}^{-1} C_{h y}^{H}$ where $C_{h y}$ is the crosscorrelation vector between the desired channel realization and the observation vector, and $C_{y y}$ is the auto-correlation matrix of the observation vector. It should be noted that to prevent aliasing, the Nyquist criterion restricts $L$ to the set $\left[2,\left\lfloor 1 /\left(2 f_{d} T_{s}\right)\right\rfloor\right]$.

\section{B. Feedback Access}

It is desirable to minimize the feedback access time because it reduces the time left for useful data transfer on the reverse channel. One approach is for all users to simultaneously transmit their quantized channel estimates to the BTS. Let $\mathbf{u}_{k}=\left[u_{k, 1}, \ldots, u_{k, b}\right]^{T}$ be the $k$ th user's feedback vector. To separate the users at the BTS, the information vectors can be spread by mutually orthogonal matrices, $W_{k} \in \mathcal{C}^{T_{r} \times b}$, where $T_{r}$ is the feedback duration. A similar approach is followed for analog feedback in [11], but in this paper we consider digital transmission for both the forward and reverse channels. The received feedback signal at the BTS is given by

$$
\mathbf{v}=\sqrt{\mathcal{P}_{r}} \sum_{k=1}^{K} g_{k} W_{k} \mathbf{u}_{k}+\mathbf{n}_{r},
$$

where $g_{k}$ is the reverse channel fading coefficient and is assumed to be constant for $T_{r}$ symbols. Furthermore, we also assume that $g_{k}$ is known to the transmitter to focus on the effects of MAI. The noise vector $\mathbf{n}_{r} \in \mathcal{C N}\left(\mathbf{0}, I_{T_{r}}\right)$ and $\mathcal{P}_{r}$ is the feedback power. It should be clear that $T_{r} \geq$ $K b$ for the matrices to be mutually orthogonal so that the feedback duration is proportional to $K$. This is true even if only one user has feedback information for the BTS [7] because user identification implies that at least $\log _{2} K$ bits must be sent. Since each user independently estimates its channel, as $K$ increases the feedback delay is dominated by the processing time at the BTS and/or the feedback transmission time. In Section IV we will consider two cases for feedback delay, namely, a constant delay assumption, and a worst-case scenario when the delay is linearly proportional to $K$.

\section{Performance Analysis}

In this section, we analyze the average BER and spectral efficiency in the presence of prediction, quantization and feedback errors. Conditioned on the predicted channel, $\hat{h}_{k}$, the true channel may be written as $h_{k}=\hat{h}_{k}+\tilde{h}_{k}$, where $\tilde{h}_{k} \sim \mathcal{C N}\left(0, \sigma_{\tilde{h}}^{2}\right)$ is the prediction error and $\sigma_{\hat{h}}^{2}=1-\sigma_{\tilde{h}}^{2}$. The instantaneous BER can be approximated as

$$
\operatorname{BER}\left(M_{i}, h, \hat{h}\right) \approx 0.2 e^{-g_{i} \mathcal{P}_{d}|h|^{2}},
$$

where $g_{i}$ is a modulation-specific constant [8], [12] and $\mathcal{P}_{d}$ is defined in (1). Let $\hat{\gamma}=|\hat{h}|^{2} / \sigma_{\hat{h}}^{2}$ be the normalized predicted SNR realization. The BER averaged over the channel for the $i$ th constellation can be written as [12]

$$
\operatorname{BER}\left(M_{i}, \hat{\gamma}\right)=E_{h} \operatorname{BER}\left(M_{i}, h, \hat{h}\right)=c_{1, i} e^{-c_{2, i} \hat{\gamma}},
$$

where

$$
c_{1, i}=\frac{0.2}{1+g_{i} \sigma_{\tilde{h}}^{2} \mathcal{P}_{d}} \quad \text { and } \quad c_{2, i}=\frac{g_{i} \sigma_{\hat{h}}^{2} \mathcal{P}_{d}}{1+g_{i} \sigma_{\tilde{h}}^{2} \mathcal{P}_{d}} .
$$


Since no power is transmitted during the outage state, the actual constant data power is given by

$$
\begin{aligned}
\mathcal{P}_{d} & =\overline{\mathcal{P}}_{d}\left(\int_{t_{1}}^{\infty} f_{\hat{\gamma}_{\max }}(y) d y\right)^{-1} \\
& =\overline{\mathcal{P}}_{d}\left(1-F_{\hat{\gamma}_{\max }}\left(t_{1}\right)\right)^{-1},
\end{aligned}
$$

where $f_{\hat{\gamma}_{\max }}(\cdot), F_{\hat{\gamma}_{\max }}(\cdot)$ are, respectively, the pdf and cumulative distribution function (cdf) of the optimal user's predicted channel. For Rayleigh fading, $f_{\hat{\gamma}_{\max }}(y)=K e^{-y}(1-$ $\left.e^{-y}\right)^{K-1}$.

By substituting (6) into (4) it can be seen that the BER for any constellation is a function of $t_{1}$. Let $\beta\left(t_{1}\right)=$ $\left(1-F_{\hat{\gamma}_{\max }}\left(t_{1}\right)\right)^{-1}$. Then, for Rayleigh fading and for $K$ users,

$$
\begin{aligned}
\beta\left(t_{1}\right) & =\left(1-\left(1-e^{-t_{1}}\right)^{K}\right)^{-1} \\
& =\left(\sum_{n=1}^{K}\left(\begin{array}{c}
K \\
n
\end{array}\right)(-1)^{n+1} e^{-n t_{1}}\right)^{-1} .
\end{aligned}
$$

Given the frame length $L$, the spectral efficiency and average BER are given by

$$
\operatorname{SE}(L)=\frac{L-1}{L} \sum_{i=1}^{N-1} R_{i}\left[F_{\hat{\gamma}_{\max }}\left(t_{i+1}\right)-F_{\hat{\gamma}_{\max }}\left(t_{i}\right)\right],
$$

and

$$
\overline{\operatorname{BER}}(L)=\frac{1}{\operatorname{SE}(L)} \sum_{i=1}^{N-1} R_{i} \int_{t_{i}}^{t_{i+1}} \operatorname{BER}\left(M_{i}, y\right) f_{\hat{\gamma}_{\max }}(y) d y,
$$

where $R_{i}=\log _{2} M_{i}$ and $(L-1) / L$ is the transmission loss due to training. The spectral efficiency is maximized with respect to the switching thresholds, and the power and bandwidth allocated for training. Thus, given a target BER constraint $\left(\mathrm{BER}_{t}\right)$ the optimization problem can be stated as

$$
\begin{aligned}
& \max _{\alpha, t_{1}, \ldots, t_{N-1}} \operatorname{SE}(L) \\
& \text { subject to } \overline{\operatorname{BER}}(L) \leq \mathrm{BER}_{t} \\
& 0 \leq t_{1}<t_{2}<\ldots<t_{N-1}<\infty, \\
& \alpha \in(0,1), L \in\left[2,\left\lfloor 1 /\left(2 f_{d} T_{s}\right)\right\rfloor\right] .
\end{aligned}
$$

Since a closed-form solution may be challenging to obtain, we use Matlab's fmincon function, which is based on sequential quadratic programming (SQP), to obtain feasible values for $\mathbf{t}=\left[t_{1}, \ldots, t_{N-1}\right]$ and $\alpha$ for each $L \in\left[2,\left\lfloor 1 /\left(2 f_{d} T_{s}\right)\right\rfloor\right]$. The optimal values are then obtained by searching over the $L$-space.

\section{A. Effect of Quantization and Feedback Errors}

Each user only needs to inform the BTS of the discrete rate it can support given a BER constraint. For a zero-error feedback channel, quantization error may cause the BTS to select a user in the same region as the maximum SNR user. However, in contrast to capacity results in [4], the spectral efficiency is not affected since discrete constellations are employed. Furthermore, we observed only a small degradation in BER performance. Thus, what is of interest is the combined effect of quantization and feedback errors. It would be shown that these imperfections cause the twin error events of erroneous user selection and erroneous constellation selection. Following the formulation of [4], let $\operatorname{Pr}\left(k_{*} \mid M_{i}\right)$ denote the probability that user $k_{*}$ is selected for transmission given that $M_{i}$ is the highest received feedback decision. It should be emphasized that reception of $M_{i}$ does not necessarily mean that the $i$ th constellation is optimal but rather that the post-detection signal, $W_{k_{*}}^{H} \mathbf{v}$, is decoded as $\mathbf{u}_{i}$. Therefore, the unconditional transmission probability for the $i$ th constellation is given by

$$
\operatorname{Pr}\left(M_{i}\right)=\sum_{j=0}^{N-1} q_{i, j}\left(F_{\hat{\gamma}}\left(t_{j+1}\right)-F_{\hat{\gamma}}\left(t_{j}\right)\right) .
$$

For the $k_{*}$ th user, its spectral efficiency for a fixed $L$ is now modified as

$$
\mathrm{SE}_{k_{*}}=\frac{L-1}{L} \sum_{i=1}^{N-1} R_{i} \operatorname{Pr}\left(k_{*} \mid M_{i}\right) \operatorname{Pr}\left(M_{i}\right) .
$$

Modifying [4, Eq. 12] the transmission probability conditioned on feedback error and $M_{i}$ is given by

$$
\begin{aligned}
\operatorname{Pr}\left(k_{*} \mid M_{i}\right) & =\sum_{k=0}^{K-1} \frac{1}{k+1}\left(\begin{array}{c}
K-1 \\
k
\end{array}\right)\left[\operatorname{Pr}\left(M_{i}\right)\right]^{k} \\
& \times\left[\operatorname{Pr}\left(\underset{l<i}{\cup} M_{l}\right)\right]^{K-k-1},
\end{aligned}
$$

where

$$
\operatorname{Pr}\left(\cup_{l<i} M_{l}\right)=\sum_{j=0}^{N-1} \sum_{l=0}^{i-1} q_{l, j}\left(F_{\hat{\gamma}}\left(t_{j+1}\right)-F_{\hat{\gamma}}\left(t_{j}\right)\right) .
$$

Using the Binomial Theorem and defining $\phi_{m, j}=\sum_{l=0}^{m} q_{l, j}$, the transmission probability becomes

$$
\begin{aligned}
& \operatorname{Pr}\left(k_{*} \mid M_{i}\right)=\frac{1}{K \sum_{j=0}^{N-1} q_{i, j}\left(F_{\hat{\gamma}}\left(t_{j+1}\right)-F_{\hat{\gamma}}\left(t_{j}\right)\right)} \\
& \times\left\{\left[\sum_{j=0}^{N-1}\left(F_{\hat{\gamma}}\left(t_{j+1}\right)-F_{\hat{\gamma}}\left(t_{j}\right)\right) \phi_{i, j}\right]^{K}\right. \\
& \left.-\left[\sum_{j=0}^{N-1}\left(F_{\hat{\gamma}}\left(t_{j+1}\right)-F_{\hat{\gamma}}\left(t_{j}\right)\right) \phi_{i-1, j}\right]^{K}\right\} .
\end{aligned}
$$

The average $\mathrm{BER}$ is given by $\overline{\mathrm{BER}}=(\mathrm{SE})^{-1} \mathrm{BER}$, where

$$
\begin{aligned}
& \mathrm{BER}^{\prime}=\sum_{i=1}^{N-1} R_{i} \operatorname{BER}\left(M_{i}, \hat{\gamma}\right) \operatorname{Pr}\left(k_{*} \mid M_{i}\right) \operatorname{Pr}\left(M_{i}\right) \\
& =\sum_{i=1}^{N-1} R_{i} \operatorname{Pr}\left(k_{*} \mid M_{i}\right) \sum_{j=0}^{N-1} \int_{t_{j}}^{t_{j+1}} \operatorname{BER}\left(M_{i}, y\right) f_{\hat{\gamma}}(y) d y .
\end{aligned}
$$

It is easy to verify by setting $q_{i, j}=\delta_{i, j}$ that (14) reduces to the case of a zero-error feedback channel. It was shown in [13] that feedback error may result in poor average BER performance in the low SNR regime. Indeed, the BER may fail to satisfy an instantaneous BER constraint. The constrained optimization of (10) allows us to capture the effect of feedback error with a single performance measure, namely the spectral efficiency. 


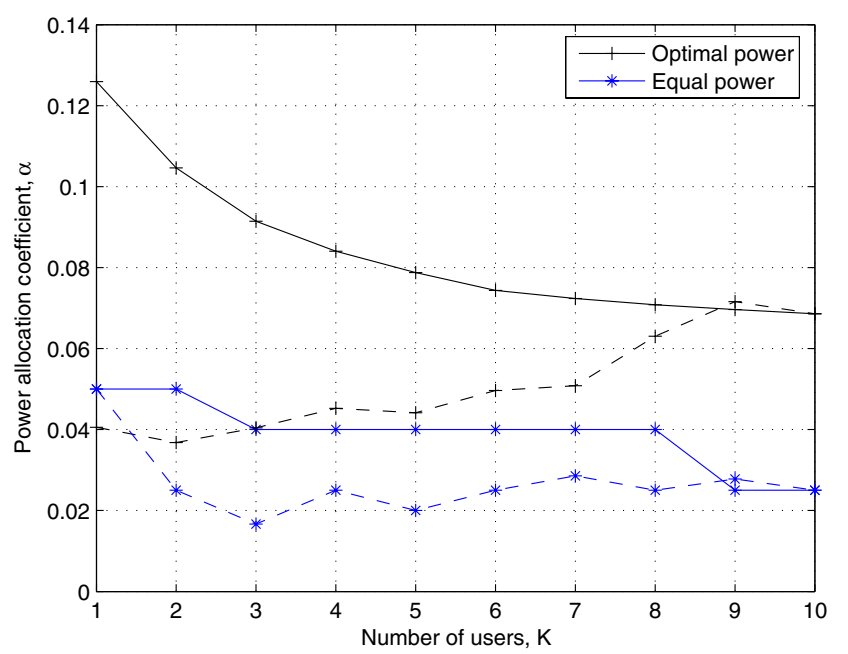

Fig. 1. Optimal and equal power allocation for a multiuser system with average $\mathrm{SNR}=20 \mathrm{~dB}$. The solid lines are for a fixed delay of $f_{d} \tau=0.2$, while the dashed lines show a worst case scenario where feedback delay is linearly proportional to the number of users.

\section{B. Feedback Error Mitigation}

The crossover probability matrix $Q$ is dependent on the feedback detection scheme employed at the BTS. Clearly, employing an error control code would lessen the impact of error compared to, for example, an uncoded maximum likelihood (ML) detection scheme. However, coding increases the amount of feedback information and it is also limited by the number of users due to the user separation requirement at the BTS. With or without coding, the channel characteristics could be used to mitigate feedback error as shown in [9], [13]. In this paper we employ a Bayesian feedback receiver [9] for error mitigation. This receiver exploits the fact that the average SNR on the forward channel depends on long-term fading, and can be assumed to be constant with respect to short-term fading. In this case, this average SNR can be reliably fed back to the BTS for adaptation to the short-term channel fading. The feedback vectors are transmitted with probability $\operatorname{Pr}\left(\mathbf{u}_{j}\right)=$ $F_{\hat{\gamma}}\left(t_{j+1}\right)-F_{\hat{\gamma}}\left(t_{j}\right)$, which depends on the average SNR of the forward channel. Since the feedback transmission probabilities are unequal, the feedback system is equivalent to a multiple hypothesis problem for which Bayesian detection is superior to ML detection. Let $C_{i j}$ denote the cost of selecting the $i$ th constellation when the $j$ th constellation is. A cost function matrix can be defined as $C_{i j}=i-j, C_{j i}=\mu C_{i j}, i>j$, for $\mu \in(0,1]$ and $0 \leq i, j, \leq N-1$. Therefore the feedback receiver mitigates feedback error by applying a penalty to the cost of making wrong decisions when such decisions would increase the BER. The probability error matrix $Q$ is obtained from numerical simulations of the feedback channel.

\section{REsults}

This section presents numerical results for the multiuser spectral efficiency when the feedback issues are considered.
The system consists of $\left\{2^{i}-\mathrm{QAM}\right\}_{i=1}^{7}$ constellations and $\mathrm{BER}_{t}=10^{-3}$. The Jakes autocorrelation function is used with a normalized Doppler frequency of $f_{d} T_{s}=10^{-3}$, while the filter size is set to a high value, $J=250$, to ensure good prediction performance as in [10]. The feedback transmission power is chosen to satisfy a feedback channel BER denoted by $q=0.01$ when ML detection is employed. First, we consider the optimal power allocation for a feedback delayconstrained system. The system is optimized according to (10) for each point on the curves. Fig. 1 shows the power allocation for optimal and equal power allocation between training and data symbols. The solid lines depict the performance for a fixed normalized feedback delay of $f_{d} \tau=0.2$. It can be seen that as the number of users increases, less power is allocated for training. In other words, for the same channel estimation error, less training power is required as the number of users increases. A different picture emerges for a worst case scenario, where feedback delay is linearly proportional to the number of users $K$. In this case the maximum delay is equal to that of the fixed delay case, i.e., $f_{d} \tau=0.2$ for $K=10$. As expected, the training power increases on average due to the increased delay with $K$. Notice that the increase in $\alpha$ is not monotonic because the performance degradation due to feedback delay is partly offset by the multiuser diversity gain. Fig. 2 shows the spectral efficiency as a function of $f_{d} \tau_{s}$, where $\tau_{s}$ is the feedback delay for a single user system. It can be seen that with increasing $K$, the performance drops sharply due to the effort required to obtain the CSI at the BTS. In other words, for a given prediction range there is a maximum number of users that can be served as indicated by the crossover points between the curves. This practical limit on $K$ may be increased by employing multiple antennas at the BTS. It is well known that multiple antennas can be used for interference cancellation and this fact has been employed for multi-antenna analog CSI feedback in [11].

The combined effect of quantization and feedback error for a zero-delay feedback channel is shown in Fig. 3. The solid lines show the spectral efficiency for $K=1,2,5,10$ users. In the low SNR region, no threshold vector $\mathbf{t}$ can be found to satisfy $\mathrm{BER}_{t}=10^{-3}$, and this implies that a rateadaptive system cannot operate at low to medium SNR due to feedback error. For the $K=1$ curve, feedback error may cause transmission of a higher level constellation even though it is more likely that a lower rate constellation (including the no transmit state) is optimal. This tends to increase the average BER, thus, to satisfy the target BER constraint, the outage region also increases. In the high SNR regime, decision errors may reduce the spectral efficiency but would not increase the average BER resulting in close to ideal performance. The same trends are true for $K>1$ but now there is the additional effect of wrong user selection, which widens the outage region as $K$ increases. It can be seen that the threshold for this outage region is $21 \mathrm{~dB}$ for $K=1,2$ and increases to $22 \mathrm{~dB}$ for $K=$ 5,10 . The dotted lines show the respective performance curves when Bayesian detection is used in lieu of ML detection. The Bayesian receiver allows for adaptive operation over most of 


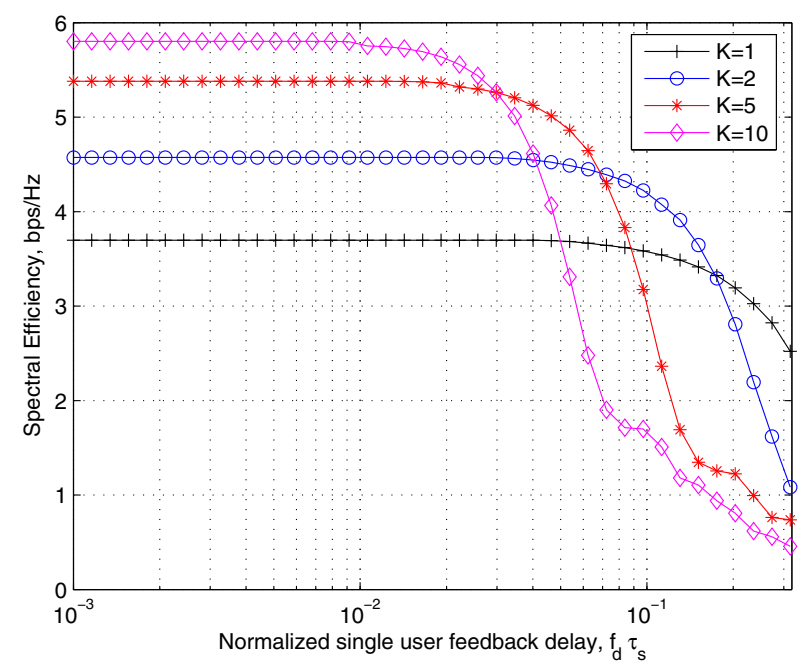

Fig. 2. Multiuser Spectral Efficiency versus normalized feedback delay for a single user and average $\mathrm{SNR}=20 \mathrm{~dB}$. The aggregate prediction range is $K \tau_{s}$.

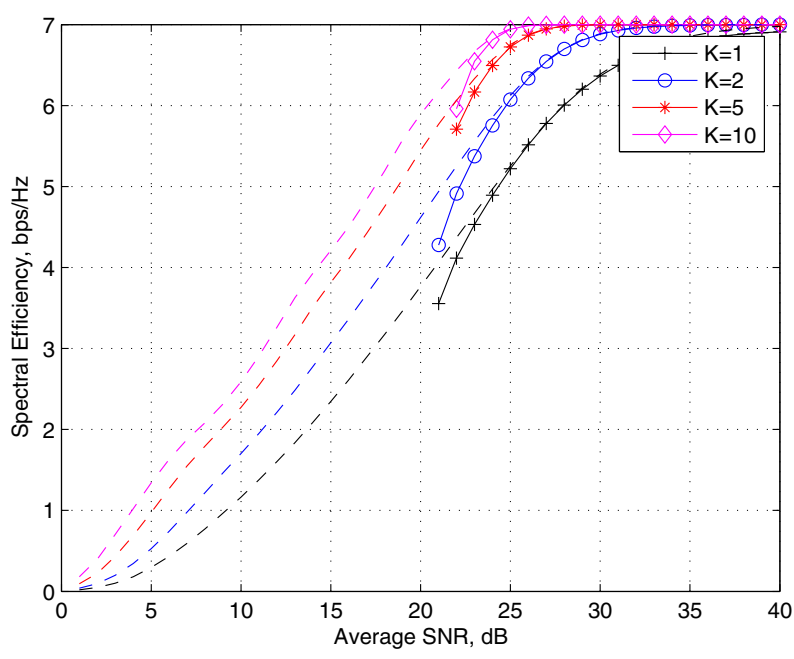

Fig. 3. Spectral Efficiency for an 8-state adaptive modulation system for ML and Bayesian feedback receivers. The system target BER is BER $_{t}=10^{-3}$ and the feedback channel quality, $q=0.01$.

the SNR range. This shows that feedback error has a more serious effect as the number of users increases because of the double effect of wrong user and wrong constellation selection due to quantization and feedback errors. Therefore, a more stringent quality of service is required for the feedback channel as the number of users increases. For example the feedback BER should be smaller, and/or reverse link power should increase with $K$.

\section{CONClusion}

The effects of prediction, quantization and feedback errors have been investigated for a rate-adaptive multiuser system. It was shown that the training power can be traded off with the number of users $K$ for a fixed feedback delay. However, user separation at the BTS implies that the feedback delay may increase proportionally with $K$, limiting the spectral efficiency growth with $K$ as the fading rate increases. Furthermore, it was shown that feedback error increases the outage SNR region as $K$ increases when the SNR thresholds are optimized to satisfy an average BER constraint. This limits the advantages of multiuser diversity gain in a practical system, and implies that feedback reliability is more critical as the number of users increases.

\section{REFERENCES}

[1] R. Knopp and P. A. Humblet, "Information capacity and power control in single-cell multiuser communications," in Proc. IEEE Int. Conf. on Comm., Seattle, WA, 1995.

[2] D. Tse, "Optimal power allocation over parallel Gaussian channels," in Proc. IEEE Int. Symposium on Information Theory, Ulm, Germany, 1997.

[3] Q. Ma and C. Tepedelenlioglu, "Practical multiuser diversity with outdated channel feedback," IEEE Trans. Veh. Technol., vol. 54, no. 4, pp. 1334-1345, July 2005 .

[4] F. Florén, O. Edfors, and B.-A. Molin, "The effect of feedback quantization on the throughput of a multiuser diversity scheme," in Proc. IEEE Globecom, San Francisco, CA, 2003.

[5] D. Gesbert and M.-S. Alouini, "How much feedback is multi-user diversity really worth?" in Proc. IEEE Intern. Conf. on Communications, Paris, France, 2004.

[6] S. Sanayei and A. Nosratinia, "Exploiting multiuser diversity with only 1-bit feedback," in Proc. IEEE Wireless Communications and Networking Conf. (WCNC), New Orleans, LA, 2005.

[7] V. Hassel, M.-S. Alouini, D. Gesbert, and G. E. Øien, "Exploiting multiuser diversity using multiple feedback thresholds," in Proc. 61st IEEE Vehicular Technology Conf., Stockholm, Sweden, Spring, 2005.

[8] S. T. Chung and A. J. Goldsmith, "Degrees of freedom in adaptive modulation: A unified view," IEEE Trans. Commun., vol. 49, pp. 15611571, September 2001.

[9] A. E. Ekpenyong and Y.-F. Huang, "Bayesian feedback detection for adaptive transmission systems," in Proc. IEEE Int. Conf. Acoustics, Speech and Signal Proc., Philadelphia, PA, 2005.

[10] X. Cai and G. B. Giannakis, "Adaptive PSAM accounting for channel estimation and prediction errors," IEEE Trans. Wireless Commun., vol. 4, no. 1, pp. 246-256, Jan. 2005.

[11] T. L. Marzetta and B. M. Hochwald, "Fast transfer of channel state information in wireless systems," IEEE Trans. Signal Processing, submitted for publication. [Online]. Available: http://mars.bell-labs.com

[12] S. Zhou and G. B. Giannakis, "How accurate channel prediction needs to be for transmit-beamforming with adaptive modulation over Rayleigh MIMO channels?" IEEE Trans. Wireless Commun., vol. 3, no. 4, pp. 1285-1294, July 2004.

[13] A. E. Ekpenyong and Y.-F. Huang, "Markov channel-based feedback schemes for adaptive modulation systems," in Proc. IEEE Globecom, Dallas, TX, 2004. 